$\mathrm{MD} / \mathrm{PhD}, 1 \mathrm{PhD})$. The two-year curriculum consists of 14 lunchtime sessions held bimonthly during academic terms. Session structures include a variety of interactive presentations, activities and facilitated discussions as well as reading material, assessment tools, and case studies. Facilitators include topic experts in academia, entrepreneurship, communications, and professional and personal development. The program is evaluated qualitatively through student satisfaction surveys after each session. RESULTS/ANTICIPATED RESULTS: Of the 2018-2019 participants, $90.8 \%$ rated the overall quality of PDC sessions as Very Good (56.05\%) or Outstanding (34.75\%). DISCUSSION/ SIGNIFICANCE OF IMPACT: Feedback indicates that the group benefited from combining predoctoral and postdoctoral trainees, although not all content was immediately relevant to early stage trainees. Trainees appreciated the opportunity to engage with experts in disciplines typically considered outside of traditional science but critical to CTS career success. The flexibility of the curriculum allowed for inclusion of timely topics, newer suites of sessions focus on the multiple dimensions of valuing your science.

4039

\section{The Development of a Mentored Writing Workgroup}

Bernadette Capili ${ }^{1}$, Bernadette Capili ${ }^{1}$, Jeanne Walker, DNP ${ }^{1}$, Barry Coller, MD ${ }^{1}$, and Kate Brown, MS, FNP ${ }^{1}$

${ }^{1}$ The Rockefeller University

OBJECTIVES/GOALS: The purpose of the mentored writing workgroup developed at The CCTS at the Rockefeller University (CCTS RU) was to promote scholarship among clinical research nurses (CRN). The goal was to publish and/or present their projects at national professional association meetings. METHODS/STUDY POPULATION: A two-part writing workshop was presented at RU in December 2018 and January 2019. Members of the RU nursing staff and CRNs from local institutions were invited to attend. Twenty-four CRNs participated in the workshops. The first workshop focused on the writing process, styles of writing and how to get started. The second concentrated on components of a manuscript, categories of papers, selection of the journal, and communications with the editorial team. After the workshops, the CRNs from RU was offered the opportunity to participate in the mentored writing workgroup. To participate, the $\mathrm{CRN}$ had to agree to attend scheduled writing meetings and submit written work for each session. The scheduled submission of written materials ensured the CRN was committed to completing their manuscripts or presentations. RESULTS/ANTICIPATED RESULTS: Three CRNs from RU participated in the writing workgroup. Two papers were accepted for publication, and one manuscript is under-review, two abstracts accepted by an international professional organization, and two presentations conducted at an area nursing school and medical center. DISCUSSION/SIGNIFICANCE OF IMPACT: A mentored writing workgroup wherein participants commit to attend writing meetings and to submit written materials in a scheduled-matter can promote scholarship. CONFLICT OF INTEREST DESCRIPTION: NA.

\section{The Entrepreneurship for Biomedicine (E4B) Training} Program

Jane M. Garbutt ${ }^{1}$, Joseph Grailer, Lillie Levin, Jessica Mozersky, Antes Schulke, Michael Kinch, and Emre Toker

${ }^{1}$ Washington University in St. Louis, Institute Of Clinical and Translational Sciences

OBJECTIVES/GOALS: Regardless of their career choices, today's biomedical researchers need to blend great science with core skills ininnovation and entrepreneurship (I\&E). The objective of this $\mathrm{NIH}$-funded education program was to develop and test a pragmatic training program to teach relevant I\&E skills. METHODS/STUDY POPULATION: We used a modified Delphi approach to identify 15 relevant competencies for I\&E and the essential topics to include in the program. Learner interviews identified preferences for online training programs (short, high-quality audio-visual content, ability to self-navigate, peer and instructor interactions). The inaugural program included 7 short, online courses that addressed how to identify and validate opportunities for innovation, sell your innovation to diverse audiences, assess its ethical consequences, work in teams, and develop resilience as an innovator. It also included mentor support, a team-based capstone project, and an optional in-person boot camp. RESULTS/ANTICIPATED RESULTS: 51 students enrolled and 41 participants from 9 institutions completed the program, including pre- and post-doctoral students and junior faculty. They organized into 10 teams to complete the capstone project, with 6 teams pitching their innovation to fellow students and mentors at the boot camp. Students rated satisfaction with courses highly overall, with $79 \%$ stating they would be disappointed if the program was no longer available. Preliminary results suggest participants increased their knowledge about and ability to perform tasks taught throughout the program. Suggestions for improvement included providing more practical advice and real-world examples to complement educational videos. DISCUSSION/SIGNIFICANCE OF IMPACT: The inaugural E4B program was well received and effective in increasing I\&E skills. Improvements will include increased opportunity for mentor interactions and for advanced entrepreneurial training. The program is open for biomedical research trainees from all institutions with a CTSA award.

4515

The Impact of First Level Training Cycles (FLTCs) on Clinical and Translational Research (CTR) in knowledge and interest in CTR of students (S) and faculty (F) from health professions and basic science programs island wide in Puerto Rico (PR)

Juan Carlos Soto Santiago ${ }^{1}$, Edgardo L. Rosado Santiago ${ }^{1}$, Efraín Flores-Rivera $^{1}$, Lizbelle De Jesus-Ojeda ${ }^{1}$, Margarita Irizarry-

Ramírez $z^{1}$, Jose Rafael Moscoso Alvarez, and Rubén García García ${ }^{1}$ ${ }^{1}$ University of Puerto Rico-Medical Sciences Campus

OBJECTIVES/GOALS: To assess the impact of FLTCs on CTR on S and $\mathrm{F}$ from health professions and basic science academic programs island wide in Puerto Rico. Cycles supported by the Title V Cooperative Project at University of Puerto Rico-Medical Sciences Campus (UPRMSC) and Universidad Central del Caribe (UCC)(Title V). METHODS/STUDY POPULATION: After offering FLTCs in CTR to $S$ and F from UPRMSC and UCC, Title V expanded it to $\mathrm{S}$ and $\mathrm{F}$ from other institutions island wide in PR. These FLTCs 
were offered the $2^{\text {nd }}$ semester of 2018 and consisted of 20 hours of interdisciplinary sessions in: introduction to and definition of CTR; preparation of a CTR-presentation; how to interview/share a presentation of a CT researcher and to prepare a research question in CTR. To assess the knowledge of S and F in the above-mentioned skills and their continuation in the $2^{\text {nd }}$ level of CTR training, surveys were administered: pre-test, at the beginning, post-test, sometime during the FLTCs, and satisfaction at the end of the FLTCs. RESULTS/ANTICIPATED RESULTS: Fifty eight (58) S/F from UPRMSC, UCC and 7 other institutions participated. Forty two $(42,72 \%)$ answered a pre-test and $31 / 42(74 \%)$ completed the post-test. Results showed that S/F: who correctly defined CTR increased from $7 \%$ to $77 \%$; their ability to identify a CT researcher increased from $10 \%$ to $83 \%$. Fifty five percent (55\%) (21/38) S/F that were certified in the FLTCs, answered the satisfaction survey. One hundred percent (100\%) indicated that the materials offered contributed in the identification of a CT researcher and a topic in CTR; $100 \%$ answered that the FLTCs contributed higher knowledge in and provided new skills in CTR. Moreover, 31/38 (82\%) S/F started the $2^{\text {nd }}$ level of training. DISCUSSION/SIGNIFICANCE OF IMPACT: The FLTCs were successful in increasing S/F knowledge of CTR and to further engage in 2 nd level of trainings. Title $\mathrm{V}$ impact extended island wide, increasing the diversity of represented health professions and science fields among participants. The interventions were deemed to be of high quality.

4235

The Use of Checklists Throughout the Lifecourse of a Clinical Research Study: The Rockefeller University Checklist Suite

Donna Brassil ${ }^{1}$, Roger Vaughan ${ }^{1}$, Arlene Hurley ${ }^{1}$, Kathleen Dowd ${ }^{1}$, Richard Hutt, and Barry S. Coller, MD ${ }^{1}$

${ }^{1}$ Rockefeller University

OBJECTIVES/GOALS: We have developed a comprehensive Translational Research Navigation Program to guide investigators all the way from protocol development through study closure. As the program evolved, we initially developed organizational tools and then restructured them into a series of checklists to ensure that critical elements were not excluded or duplicated. METHODS/STUDY POPULATION: A series of checklists to assure that all research elements, including regulatory, scientific, and institutional, are addressed from protocol inception through study closure were developed by clinical research coordinators/navigators. The checklists are periodically updated and modified to reflect changing local and national regulations and policies. The first tool became the "Protocol Development Checklist" and then additional tools were developed and modified into a suite of navigation checklists that include "Protocol Implementation Checklist," "Protocol Conduct Checklist," and "Protocol Completion Checklist." RESULTS/ANTICIPATED RESULTS: The checklists have been incorporated into the Translational Research Navigation Program and have enhanced the organization and quality of protocols throughout their lifespan. For example, implementation of the Protocol Development Checklist resulted in a reduction in time to IRB approval (currently 10 days), and implementation of the Protocol Implementation Checklist has impacted the time from IRB approval to study start-up. The Protocol Conduct Checklist has aided investigators in being better prepared and more organized for study conduct activities and the Protocol Closure Checklist has assured timely protocol closure and regulatory compliance, including reporting to ClinicalTrials.gov. DISCUSSION/SIGNIFICANCE OF IMPACT:
Protocol checklists are powerful tools to enhance thoroughness, organization, and quality of the clinical research process. The Rockefeller University protocol checklists are available to the CTSA and Scientific Communities. CONFLICT OF INTEREST DESCRIPTION: NA.

4274

Thirteen Years of Pipeline Programming at the University of Rochester's Clinical \& Translational Science Institute to Train Physician-Scientists

Alaina Maiorano ${ }^{1}$, Edwin van Wijngaarden ${ }^{1}$, Alfred Vitale ${ }^{1}$, Timothy De Ver Dye ${ }^{1}$, Robert Gross ${ }^{1}$, and Kerry O'Banion ${ }^{1}$

${ }^{1}$ University of Rochester Medical Center

OBJECTIVES/GOALS: Physician-scientists play a vital role in biomedical research but this chosen career path has many challenges, such as long training periods and funding. The University of Rochester (UR) CTSI pipeline programs address this by enabling medical trainees to partake in enriched research experiences. METHODS/STUDY POPULATION: The UR CTSI TL1 is a training grant from the National Center for Advancing Translational Science (NCATS), which funds predoctoral trainees. The TL1-funded physician-scientist pipeline includes the Academic Research Track (ART) year-out program and the Medical Science Training Program (MSTP). We describe the characteristics and training outcomes of TL1-funded trainees. We also obtained testimonials of current and former trainees regarding their career component decision-making, and their perception of programs, in order to identify how best to address the challenges of the physician-scientist workforce, and to facilitate the transition between the clinic and bench. RESULTS/ANTICIPATED RESULTS: From 2006-2019, the UR CTSI has had 56 ART trainees and 17 MSTP trainees complete training; six trainees have transitioned into the MSTP after completing the ART program. As of 2019, 63 of 67 graduated trainees (94\%) have continued their engagement in CTS after graduation. Importantly, our programs have facilitated the careers of 31 women (39.7\%) and 12 under-represented minorities (15.4\%). We will present a breadth of qualitative data to inform which parts of the TL1-related programs have been successful, and which parts could use programmatic improvement to aid the transition into the physician-scientist workforce. DISCUSSION/SIGNIFICANCE OF IMPACT: Physicianscientist training barriers in the US have resulted in a shortage of these professionals in the clinical and translation workforce. Our data show the UR CTSI has been successful in addressing several of these challenges via the TL1-funded ART, MSTP, and ART/MSTP dual program pipeline.

\section{Evaluation}

4124

\author{
An innovative Tool for Completing the Clinical and \\ Translational Science Award (CTSA) Research \\ Performance Progress Report (RPPR) using REDCap \\ Maran Subramain ${ }^{1}$, DeAnna O'Quinn ${ }^{1}$, and Heath Davis ${ }^{2}$ \\ ${ }^{1}$ University Of lowa Institute for Clinical and Translational Science; \\ 2University of lowa
}

OBJECTIVES/GOALS: The RPPR Tool was created to accurately and systematically track our CTSA's overall program goals and core's 